\title{
Diffusion in normal and critical transient chaos
}

\author{
Z. Kaufmann ${ }^{1}$, H. Lustfeld ${ }^{2}$, A. Németh ${ }^{1,3}$ and P. Szépfalusy ${ }^{1,4}$ \\ ${ }^{1}$ Institute for Solid State Physics, Eötvös University, Múzeum krt. 6-8, H-1088 Budapest, Hungary \\ ${ }^{2}$ Institut für Festkörperforschung, Forschungszentrum Jülich, D 52425 Jülich, Germany \\ ${ }^{3}$ Brody Research Center, GE Lighting Tungsram Co. Ltd., Váci út 77, H-1340 Budapest, Hungary \\ ${ }^{4}$ Research Institute for Solid State Physics, P.O. Box 49, H-1525 Budapest, Hungary
}

In this paper we investigate deterministic diffusion in systems which are spatially extended in certain directions but are restricted in size and open in other directions, consequently particles can escape. We introduce besides the diffusion coefficient $D$ on the chaotic repeller a coefficient $\hat{D}$ which measures the broadening of the distribution of trajectories during the transient chaotic motion. Both coefficients are explicitly computed for one-dimensional models, and they are found to be different in most cases. We show furthermore that a jump develops in both of the coefficients for most of the initial distributions when we approach the critical borderline where the escape rate equals the Liapunov exponent of a periodic orbit.

\section{INTRODUCTION}

Diffusion is one of the most common and most important phenomena of nature. In physics it is important for nondeterministic systems, governed by noise, for quantum systems and for deterministic systems, as well [1.,2, 3, $4,5,5,6,7,8,8,97$. In this paper we want to investigate transient diffusion in deterministic systems. By this we mean diffusion in a system spatially extended in certain directions which is however not closed in other directions and particles can escape. The diffusion is usually defined in the limit of infinitely long times. However, the intrinsic feature of transient chaos (see for a review [10, 11]) is the limited time a system is close to a chaotic repeller. Only during this time the system shows the characteristics we are interested in. One can have in mind, for instance, a channel in a two-dimensional mesoscopic system containing Lorentz-type scatterers which is infinite in one direction but has a finite width in the transverse direction. Sooner or later all particles are scattered away from the channel and get lost if we assume free boundaries. During their motion in the channel the particles are scattered and can exhibit diffusion in the extended direction. So the question arises in which way this diffusion should be characterized.

The basic problem is how to define the diffusion coefficient. One possibility is to compute the diffusion coefficient $D$ for particles on the repeller. That requires the computation of the diffusion coefficient for trajectories of those particles staying for ever in the channel. Following this line we can go to the limit of infinite time and thus can proceed as in the permanent chaos case. This formulation, however, can not provide a full description, since the probability is zero that a trajectory is on the repeller. A quite different view is to compute the actual properties in the neighborhood of the repeller, which is the main goal of the present paper.

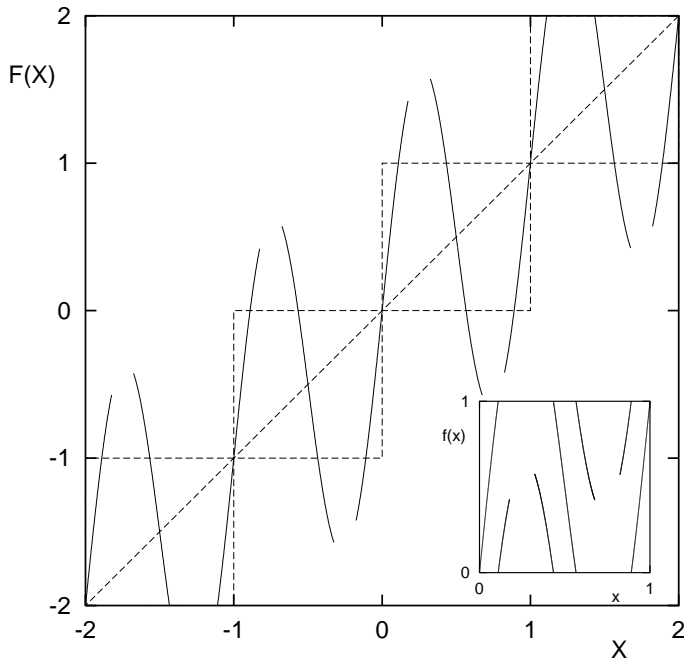

FIG. 1. An example for map $F$ with translational symmetry and escape. The inset shows the corresponding reduced $\operatorname{map} f$.

To avoid complications with continuous time and higher dimensions we discuss here one-dimensional discrete maps defined on the real line. The extension of our formulation to more general cases is straightforward. The map $X_{t+1}=F\left(X_{t}\right)$ has translational symmetry $F(x+1)=F(x)+1$ and is not defined for a periodic set of intervals, called in the following windows, from which the trajectory escapes (Fig. 1). It is assumed that the map is chaotic in the sense that there exists no stable periodic orbit. Such maps can be described by reduced maps defined in $\mathcal{U}=[0,1]$ [4, 5,6$]$. Let us denote by $N$ the box of the interval $[N, N+1$ ). A phase point (called particle in the following) being after $t$ iterations in box $N$ with position $X_{t}$ can either remain in that box or move to one of the nearby boxes or get lost. Which of these possibilities occurs depends only on fractional part $\{X\}$ of $X$, since using the new variables $N=[X], x=\{X\}$ 
we get a dynamical system of the two variables

$$
x_{t+1}=f\left(x_{t}\right), \quad N_{t+1}=N_{t}+\Delta\left(x_{t}\right),
$$

which is equivalent to the map $F$. Here $f(x)=\{F(x)\}$ is the reduced map (cf. Fig. 1), which has a window where trajectories escape, and the integer part $\Delta(x)=[F(x)]$ determines the steps among the boxes. We call a system with escape open, otherwise closed. The reduced maps with escape are generalizations of those introduced in [4,5,6] and have been studied also in a different context [12.

Let us follow the reduced trajectories of particles in the interval $\mathcal{U}$. At the beginning the distribution of the initial points $x_{0}$ is according to an arbitrary density $P_{0}$ in $\mathcal{U}$. The change of $P_{t}$ after each step of iteration is induced by $f$ alone and given by the Frobenius-Perron operator $P_{t+1}(x)=\mathcal{L} P_{t}(x) \equiv \sum_{z: f(z)=x} P_{t}(z) /\left|f^{\prime}(z)\right|$. Repeated application of $\mathcal{L}$ leads to an average decrease of the density and to a change of its structure at the same time. Finally a quasistationary state is reached: the density normalized in $\mathcal{U}$ after each step has converged to $P$, the density of the conditionally invariant measure [13], whereas the density itself is decreasing after each iteration by a certain rate, related to the escape rate $\kappa$ :

$$
\mathcal{L} P=\lambda_{c} P, \quad\left|\ln \lambda_{c}\right|=\kappa .
$$

This emphasizes the importance of the quasistationary state. Computing the properties of that state corresponds to taking the limit of large times $T$ to reach it. We can define a coefficient $\hat{D}$ in the quasistationary state, which is analogous to a usual diffusion coefficient in the sense that it measures the mean quadratic deviation of the trajectories from their average shift. We call $\hat{D}$ the transient diffusion coefficient. In general $\hat{D}$ differs from $D$ as will be shown explicitly for 1D models.

We study the properties of both diffusion coefficients as a function of a control parameter while keeping the escape rate constant. Within the family of such maps there is a limit when the Liapunov exponent of one of the unstable periodic orbits has reached the value of the escape rate and still the conditionally invariant measure has a density [14]. This situation is called the critical borderline of transient chaos 14,15]. We show that here both $D$ and $\hat{D}$ can have two different values depending on the initial distribution of trajectories. This will be shown to be traced back to the existence of two conditionally invariant densities with different escape rates.

In Section 2 we derive the diffusion coefficient on a repeller, $D$, and the transient diffusion coefficient $\hat{D}$. Then we choose maps $F$ of open systems whose reduced maps are in the family of fully developed chaotic maps [16,14. We present results that clearly demonstrate the difference between $D$ and $\hat{D}$. In Section 3 we show that on approaching the critical borderline of transient chaos a jump develops in both $D$ and $\hat{D}$ for most of the initial distributions. The conclusion ends the paper.

\section{THE DIFFUSION COEFFICIENTS OF TRANSIENT CHAOS}

To get the diffusion coefficient for open systems we have to know the shift $S_{t}$ of particles among the boxes at a given time

$$
S_{t}=N_{t}-N_{0}=\sum_{k=0}^{t-1} \Delta\left(x_{k}\right)
$$

Let us compute the average value of $S_{t}$ first by averaging over an initial distribution $P_{0}\left(x_{0}\right)$ of $x_{0}$ values with $f^{t}\left(x_{0}\right)$ in $\mathcal{U}$. Thus we obtain $\left\langle S_{t}\right\rangle_{0}=\sum_{l=0}^{t-1} \int_{\mathcal{U}_{t}} \Delta\left(f^{l}(x)\right) P_{0}(x) d x / \int_{\mathcal{U}_{t}} P_{0}(x) d x$ with $\mathcal{U}_{t}=f^{-t}(\mathcal{U}) \cap \mathcal{U}$. For $t \rightarrow \infty \mathcal{U}_{t}$ becomes a repelling invariant set of the reduced map which will be called repeller in the following. To get the diffusion coefficient the procedure is to take the limit $t \rightarrow \infty$, which restricts the trajectories on the repeller. Apart from that the derivation of the corresponding expressions becomes straightforward along the lines of permanent diffusion [5],6]. We use the Frobenius-Perron operator $\mathcal{L}$ to write $\left\langle S_{t}\right\rangle_{0}=\sum_{l=0}^{t-1} \int_{\mathcal{U}}\left[\mathcal{L}^{t-l} \Delta \mathcal{L}^{l} P_{0}\right](x) d x / \int_{\mathcal{U}}\left[\mathcal{L}^{t} P_{0}\right](x) d x$. But $\lim _{l \rightarrow \infty} \lambda_{c}^{-l} \mathcal{L}^{l}$ acts as a projector on $P$, the density of the conditionally invariant measure. Thus the result for the averaged drift is

$$
\lim _{t \rightarrow \infty} \frac{1}{t}\left\langle S_{t}\right\rangle_{0}=\int_{\mathcal{U}} \Delta(x) \mu(d x) \equiv\langle\Delta\rangle_{\mu},
$$

where $\int_{\mathcal{U}} \mu(d x)=1$ and $\mu$ is the natural invariant measure on the repeller. $\left\langle S_{t}^{2}\right\rangle_{0}$ can be computed in an analogous manner. The diffusion coefficient is then defined by

$$
D=\lim _{t \rightarrow \infty} \frac{\left\langle\left(S_{t}-\left\langle S_{t}\right\rangle_{0}\right)^{2}\right\rangle_{0}}{2 t}
$$

which can be written in terms of the correlation function $C_{\Delta \Delta}$ on the repeller as

$$
D=\lim _{t \rightarrow \infty} \frac{1}{2 t} \sum_{l, m=0}^{t-1} C_{\Delta \Delta}(|l-m|),
$$

where $C_{\Delta \Delta}(l)=\left\langle\left(\Delta\left(f^{l}(x)\right)-\langle\Delta\rangle_{\mu}\right) \Delta(x)\right\rangle_{\mu}$. The expressions are completely analogous to the formulae of closed systems [5, 6]. In spite of the analogies $\langle\Delta\rangle_{\mu}$ and $D$ cannot be accepted as the only quantities characterizing open systems since a trajectory moves on the repeller with probability zero.

Because of this a second procedure is suggested. Let us investigate particles that have been injected into the system and have survived (i.e. have not escaped) after a time $T$ long enough to have the density $P_{T}(x)$ of the reduced distribution of these particles close to the density $P(x)$ of the conditionally invariant measure. We consider 
the shift of the particles from time $T$ until their escape $S_{e}\left(x_{T}\right)=N_{T+\tau\left(x_{T}\right)}-N_{T}=\sum_{k=0}^{\tau\left(x_{T}\right)-1} \Delta\left(x_{T+k}\right)$, where $\tau(x)$ is the number of steps taken by the trajectory of $x$, i.e. $f^{\tau}(x) \in \mathcal{U} \equiv[0,1]$ and $f^{\tau(x)+1}(x) \notin \mathcal{U}$. The average of this shift $\left\langle S_{e}\right\rangle_{T}=\int_{\mathcal{U}} S_{e}(x) P_{T}(x) d x$ over the trajectories considered and the average lifetime $\langle\tau\rangle_{T}=$ $\int_{\mathcal{U}} \tau(x) P_{T}(x) d x$ can be evaluated in the limit $T \rightarrow \infty$ using properties of the Frobenius-Perron operator. The result is $\langle\tau\rangle=\lambda_{c} /\left(1-\lambda_{c}\right)$ and an average drift

$$
\frac{\left\langle S_{e}\right\rangle}{\langle\tau\rangle}=\langle\Delta\rangle \equiv \frac{1}{\lambda_{c}} \int_{\mathcal{U}_{1}} \Delta(x) P(x) d x .
$$

For sake of simplicity the $\infty$ index at the sign of average is omitted.

We can characterize the transient spreading of the particles by the transient diffusion coefficient defined as

$$
\hat{D}=\lim _{T \rightarrow \infty} \frac{\left\langle\hat{S}_{e}^{2}\right\rangle_{T}}{2\langle\tau\rangle_{T}}=\frac{\left\langle\hat{S}_{e}^{2}\right\rangle}{2\langle\tau\rangle},
$$

where $\hat{S}_{e}\left(x_{T}\right)=\sum_{k=0}^{\tau\left(x_{T}\right)-1}\left(\Delta\left(x_{T+k}\right)-\langle\Delta\rangle\right)$, the corrected shift is introduced. Through steps similar to those which led to Eq. (7) $\hat{D}$ can be related to the correlation function of transient chaos [15] $c_{\Delta \Delta}(n)=$ $\lambda_{c}^{-(n+1)} \int_{\mathcal{U}_{n+1}}\left(\Delta\left(f^{n}(x)\right)-\langle\Delta\rangle\right) \Delta(x) P(x) d x$ by the expression

$$
\hat{D}=\sum_{n=-\infty}^{\infty} \lambda_{c}^{|n|} c_{\Delta \Delta}(|n|) .
$$

Physically this means the following. After injection drift and spreading of the injected particles depend on the initial distribution, but its influence decreases exponentially fast. After a long time $T$ it is no longer important and then further drift and spreading of the particles is dictated by the properties of the open system alone, characterized by $\left\langle S_{e}\right\rangle$ and $\hat{D}$, respectively. This general picture has to be refined, however, if the system has more than one smooth conditionally invariant measure as will be seen later.

In the numerical calculations of open systems we take the reduced map $f$ from the family of maps defined on the interval $[0,1]$ and given by the inverse branches $f_{l}^{-1}(x)=$ $(x+v(x)) / 2 R$ (lower branch), $f_{u}^{-1}(x)=1-f_{l}^{-1}(x)$ (upper branch), where $R \geq 1, v(0)=0, v(x)$ is symmetric and $\left|v^{\prime}(x)\right| \leq 1$. The density of a conditionally invariant measure can be expressed as

$$
P(x)=1+v^{\prime}(x) .
$$

The width of the window and the escape rate are given by $1-R^{-1}$ and $\kappa=\ln R$, respectively. Note that in the limit $v \equiv 0$ the open tent map emerges whereas in the limit $v^{\prime}(0)=1$ we reach the state what we call critical borderline of transient chaos, since the Liapunov exponent of the fixed point at the origin equals the escape rate 14,15 .
In our numerical computations we take $\mathcal{U}=[0,1]$ and we use the piecewise parabolic map [14,16] which corresponds to $v(x)=d \cdot x(1-x),-1 \leq d \leq 1$. We require, that a particle hitting the left (right) branch of $f$ hops to the left (right) adjacent box $N$ in the map $F$. If it hits neither branch then it escapes. This yields $\Delta(x)=-1$ if $x<1 /(2 R)$ and 1 if $x \geq 1-1 /(2 R)$. To be distinctively in the repeller regime we choose $R=1.5$. However, the computation of $D$ is not trivial in that regime. Reliable results were obtained only after we had applied backward iteration up to a depth of 20 steps, left out the corresponding gaps of the repeller and had started with $10^{8}$ trajectories $\left(10^{9}\right.$ in case of $\left.d=0.95\right)$. While computing $\hat{D}$ we did not take into account the first 20 steps $(T=20)$ to ensure the quasistationarity of the system. In Fig. 2 the diffusion coefficients $D$ and $\hat{D}$ are shown for $d$ values ranging from 0 to 1 . Whereas they agree for the tent map a difference between $D$ and $\hat{D}$ develops when the parameter $d$ increases. The two extra points for $d=1$ in Fig. 2 will be explained in the next section.

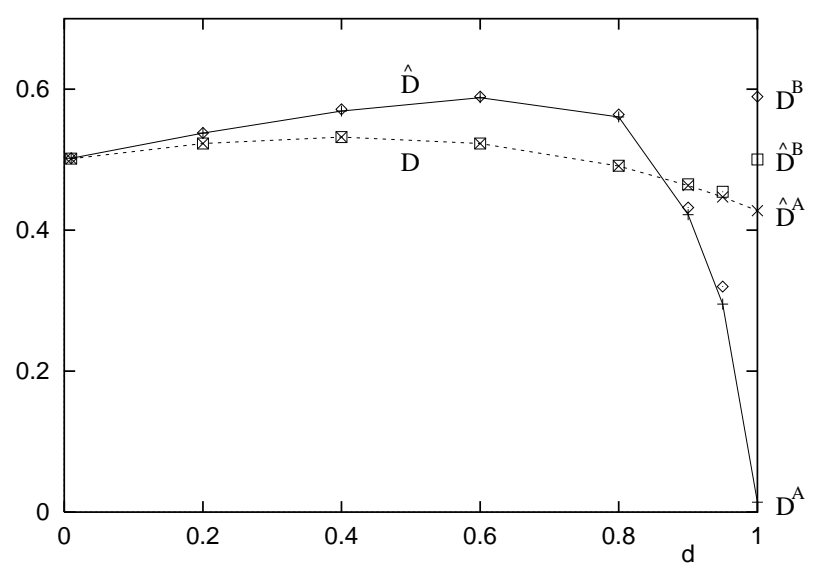

FIG. 2. Numerical results for $D$ and $\hat{D}$ in function of $d$. The superscript refers to the choice of the interval on which the initial points were distributed.

\section{DIFFUSION IN CRITICAL TRANSIENT CHAOS}

A peculiar situation emerges when we reach the critical borderline of transient chaos $(d=1$ in the example treated). Eq. (10) is still valid and gives a density $P^{A}(x)$ (equal to $2(1-x)$ in case of our example) for a conditional invariant measure with an escape rate $\kappa^{A}=\ln R$. The reason for introducing the superscript $A$ is explained below. The corresponding eigenfunction $P^{+A}$ of the adjoint Frobenius-Perron operator consists of two $\delta$-function peaks $P^{+A}=(\delta(x)+\delta(1-x)) / 2[15]$. This makes possible the existence of an other conditionally invariant measure with density. To find it one should iterate a function $\phi_{0}$ that is positive everywhere in $(0,1)$ and $\phi_{0}(0)=\phi_{0}(1)=0$ by the Frobenius-Perron operator with the prescription $\phi_{t+1}=\alpha_{t} \mathcal{L} \phi_{t}$, where $\alpha_{t}$ is chosen in such a way that the norm of $\phi_{t+1}$ is kept to unity. The 
iterated functions remain orthogonal to $P^{+A}$ because in the critical state the derivative of the map at the maximum points is infinity. Furthermore it is easy to see that the positivity of $\phi_{t}$ is also maintained. In the limit $t \rightarrow \infty$ the iteration leads to a nonnegative eigenfunction which can be taken as the density $P^{B}(x)$ of a conditionally invariant measure. The obtained eigenvalue $\lambda_{c}^{B}$ defines a new escape rate $\kappa_{B}=\left|\ln \lambda_{c}^{B}\right|$.

This means that we have two different conditionally invariant measures with different escape rates in the critical state. Which of them is obtained in the long time limit when iterating the Frobenius-Perron operator depends on the initial density $P_{0}(x)$. It is clear from the above considerations that starting with a smooth $P_{0}(x)$ such that $P_{0}(0)=P_{0}(1)=0$ we reach $P^{B}(x)$, otherwise $P^{A}(x)$. When simulating the initial density by a large number of initial points the obtained distribution $P_{T}(x)$ of iterates at a large time $T$ is governed by the same rule. Note that to balance the escape we have to increase the number of initial points when increasing $T$.

The above rule for the asymptotic distribution is reflected in the values of the diffusion coefficients $D$ and $\hat{D}$. In the numerical calculations we distributed a large number of particles uniformly on intervals, $[0,1]$ and $[0.1,0.2]$, to simulate initial densities in the basin of attraction of $P^{A}$ and $P^{B}$, respectively. It is clear from Fig. $2($ at $d=1)$ that the result depends strongly on the initial distribution (as well as values of the escape rate and the average drifts $\langle\Delta\rangle_{\mu},\langle\Delta\rangle$, not displayed here). Note, the value of $D^{A}$ is zero due to the fact that the natural measure induced by $P^{A}$ on the repeller (but not the repeller itself) degenerates, it becomes a Dirac delta function at the origin 14, 15.

\section{DISCUSSION}

In this paper we have investigated statistical properties in long time limit of trajectories in the transiently chaotic regime by the help of two diffusion coefficients $D$ and $\hat{D}$. For $D$ this time limit refers to the whole length of the trajectories (a procedure leading automatically to experience the properties of the repeller itself). In contrast the long time limit of $\hat{D}$ is only needed to measure it in the quasistationary state. Though the definition of a transient diffusion constant as introduced in the present paper seems to be the most natural one, it is not the only possibility. In future work we plan to examine also alternative definitions.

We have shown furthermore that a map at the critical borderline of transient chaos has two smooth conditionally invariant measures. We find, however, that in nearly all practical situations (i.e. when the initial phase points are concentrated in the inner part of the interval) the relevant measure is not the usual one, belonging to the smaller escape rate, but an other eigenfunction of the Frobenius-Perron operator $\mathcal{L}$.
In this paper we have treated a 1D model which can be conceived as describing the dynamics along the expanding direction of a $2 \mathrm{D}$ map. It is expected that a more complete treatment would not change the conclusions. We plan as a next step to study 2D Hamiltonian systems.

\section{ACKNOWLEDGMENTS}

This work has been supported in part by the GermanHungarian Scientific and Technological Cooperation Investigation of classical and quantum chaos, by the Hungarian National Scientific Research Foundation under Grant Nos. OTKA T017493 and OTKA F17166, the USHungarian Science and Technology Joint Fund in cooperation with the NSF and the Hungarian Academy of Sciences under project No. 286, and the Ministry of Education of Hungary under grant No. MKM337. One of the authors (H. L.) would like to thank Prof. P. Szépfalusy for the hospitality at Eötvös University where this work has been done.

[1] H. Haken, Advanced Synergetics, Springer 1983.

[2] N. W. Ashcroft and N. D. Mermin, Solid State Physics, Holt Rinehart and Winston 1976.

[3] R. Artuso, Physica D 76, 1 (1994) and references therein.

[4] T. Geisel and J. Nierwetberg, Phys. Rev. Lett. 48, 7 (1982), T. Geisel and J. Nierwetberg, Z. Phys. B 56, 59 (1984).

[5] M. Schell, S. Fraser and R. Kapral, Phys. Rev. A 26, 504 (1982).

[6] S. Grossmann and H. Fujisaka, Phys. Rev. A 26, 1179 (1982).

[7] P. Gaspard, G. Nicolis, Phys. Rev. Lett. 65, 1693 (1990).

[8] P. Cvitanovic, J. P. Eckmann, P. Gaspard, Chaos, Solitons and Fractals 6, 113 (1995).

[9] T. Tél, J. Vollmer and W. Breymann, Europhys. Lett. 35, 659 (1996).

[10] T. Tél, in Directions in Chaos, Vol. 3, edited by Hao Bailin (World Scientific, 1990), pp. 149-211; and in STATPHYS 19, The proceedings of the 19th IUPAP Conference on Statistical Physics, edited by Hao Bai-Lin (World Scientific, 1996), pp. 346-362.

[11] A. Csordás, G. Györgyi, P. Szépfalusy and T. Tél, Chaos 3, 31 (1993).

[12] R. Mainieri, Physica D 83, 206 (1995).

[13] G. Pianigiani and J. A. Yorke, Trans. Am. Math. Soc. 252, 351 (1979); G. Pianigiani, J. Math. Anal. Appl. 82, 75 (1981).

[14] A. Németh and P. Szépfalusy, Phys. Rev. E 52, 1544 (1995).

[15] H. Lustfeld and P. Szépfalusy, Phys. Rev. E 53, 5882 (1996).

[16] G. Györgyi and P. Szépfalusy, Z. Phys. B 55, 179 (1984). 